\title{
Contrast Enhanced Ultrasound in CT-undetermined Focal Liver Lesions
}

Authors

Affiliations
S. W. Sandrose ${ }^{1}$, S. Karstrup ${ }^{2}$, O. Gerke ${ }^{3}$, S. Rafaelsen

Affiliation addresses are listed at the end of the article
Key words

- CEUS

- CT

sensitivity

- specificity

ultrasound received 10.03.2016 accepted $\quad 20.10 .2016$

Bibliography

DOI http://dx.doi.org/

10.1055/s-0042-120272

Ultrasound International Open

2016; 2: E129-E135

(c) Georg Thieme Verlag KG

Stuttgart · New York

ISSN 2199-7152

Correspondence

S. W. Sandrose, Master of Medical Imaging MMDI

Faculty of Health and

Technology

Metropolitan University College

Sigurdsgade 26

2200 Copenhagen $\mathrm{N}$

Denmark

Tel.: + 45/516 32562

sesa@phmetropol.dk

\section{Abstract}

$\nabla$

Purpose: The purpose of this study was to examine the diagnostic accuracy of CEUS in the diagnosis of focal liver lesions, which were undetermined at CT scan.

Materials and Methods: From January 2010 to December 2010, patients with CT-undetermined focal liver lesions were included in this study. A total of 78 patients were evaluated: 41 men and 37 women, mean age 61.8 years; age range 30-91 years. All patients were examined with GE LOGIQ $9 \mathrm{E}$ ultrasound scanners with contrast-specific software, and SonoVue intravenous bolus. The standard of reference was composite consisting of: percutaneous biopsy, surgical resection, PET/ CT and clinical follow-up.

Results: The 78 included patients had 163 undetermined focal liver lesions, mean size $1.1 \mathrm{~cm}$, range $0.1-5.3 \mathrm{~cm}$. There were 18 malignant and 145 benign liver lesions, as defined

\section{Introduction}

\section{$\nabla$}

Detection and characterisation of benign and malignant focal liver lesions (FLLs), often as incidental findings, are a common radiological task. Volk et al. [1] reported incidental FLLs in $6 \%$ of 1892 patients scanned by computed tomography (CT).

FLLs are commonly found in patients with and without cancer, and have been reported to be present in up to $50 \%$ of autopsies [2]. Liver metastases are found in $25-50 \%$ of patients with nonhaematological malignant conditions, often in colorectal cancer, lung cancer, breast cancer and pancreatic cancer [3]. Approximately $25-50 \%$ of small $(<2 \mathrm{~cm})$ FLLs in oncological patients are of a benign nature [4].

Cysts are the most commonly found FLLs, and CT, in contrast to ultrasound, has difficulties differ- by the standard of reference. In differentiating between benign vs. malignant CEUS demonstrated sensitivity, specificity, PPV, NPV and accuracy of $94.4 \%$ (95\% CI: 56.3-99.5\%), 99.3\% (95\% CI: 94.9-99.9\%), 94.4\% (95\% CI: 56.399.5\%), 99.3\% (95\% CI: 94.9-99.9\%) and 98.7\% (95\% CI: 94.9-99.7\%), respectively. If the CEUSinconclusive results were assumed to indicate malignancy, then sensitivity, specificity, PPV, NPV and accuracy would be $95.8 \%$ (95\% CI: 66.499.6\%), 98.6\% (95\% CI: 94.4-99.7\%), 92.0\% (95CI: 65.1-98.6\%), 99.3\% (95\% CI: 95.0-99.9\%), 98.2\% (95\% CI: 94.4-99.5\%).

Conclusion: The results of this study showed a high diagnostic accuracy for CEUS in undetermined focal liver lesions found by CT. Due to a high diagnostic accuracy of CEUS in this study, it was cautiously concluded that CEUS is useful in differentiating between malignant and benign focal liver lesions in clinical practice.

entiating small cysts from malignant lesions, due to partial volume [5]. Hemangiomas are the most common solid focal liver lesions with a prevalence of $7-21 \%$. Focal nodular hyperplasia (FNH) is the second most commonly occurring benign focal liver lesion, with a prevalence of $3 \%$ [4]. Other benign focal liver lesions are adenomas and abscesses. Focal fatty sparing and focal fatty infiltration are also commonly found in the liver. Even though they are not focal lesions, they can represent pseudo lesions on CT or ultrasound; also the infrequent third inflow can represent a pseudo lesion on CT [4].

Ultrasound has a low sensitivity in diagnosis of FLLs in comparison with other diagnostic modalities such as CT [4]. Patients are therefore usually examined with $\mathrm{CT}$ or magnetic resonance imaging (MRI) [4]. Contrast agents used in CT and MRI are known to be nephrotoxic and are contraindi- 
cated in patients with renal insufficiency and present a risk of inducing contrast-nephropathy [6]. CT is more expensive and requires more resources than contrast-enhanced ultrasound (CEUS). Another disadvantage of CT is its use of ionizing radiation [6]. MRI is contraindicative in patients with claustrophobia and patients with pacemakers or metal implants [6]. SonoVue is considered safe to use in patients with renal insufficiency, because SonoVue is not nephrotoxic [6].

The diagnostic advantages of CEUS in the diagnosis of FLLs have been reported in the literature, and the use of CEUS is recommended in undetermined CT findings $[7,8]$. Studies have shown that CEUS has a sensitivity of $85-90 \%$ and a specificity of $86-90 \%$, in differentiating between benign and malignant FLLs [7,9-11]. Sensitivity and specificity of CEUS in CT-undetermined FLLs have not been fully studied, and have only been shown useful in a few studies $[11,12]$. In addition, the clustered nature of the liver lesions has not been taken into account in the previous studies.

An accurate and safe diagnostic method with few side effects is essential for satisfactory patient outcome and management. Therefore, it is of clinical importance to have a high diagnostic accuracy of CEUS in CT-undetermined FLLs.

The purpose of this study was to examine the diagnostic accuracy of CEUS in the diagnosis of focal liver lesions, which were undetermined in the initial CT scan.

\section{Materials and Methods}

$\nabla$

The design of this study follows the Standards for Reporting of Diagnostic Accuracy (STARD) in order to achieve the methodological quality that is required in the reporting of diagnostic accuracy [13]. The study was approved by the National Data Protection Agency (journal nr. 2013-41-1928). Patient lists were obtained through the Radiology Information System (RIS) and Picture Archiving and Communications System (PACS).

\section{Population}

This study retrospectively examined all CT studies performed from January 2010 to December $2010(n=920)$ by a single centre. Patients with at least one CT-undetermined FLL who were also referred to CEUS were included $(n=84) .3$ patients with 5 FLLs were excluded because CEUS was not performed for various reasons, such as patients not showing up or being unwilling to do the exam with contrast. 3 patients with 7 FLLs were inconclusive on CEUS and were excluded from the primary analyses, but included in the sensitivity analyses. Thus 78 patients with 163 FLLs, 41 men and 37 women, with a mean age 61.8 years (age range 30-91, SD 14.2), comprised the study population. The inclusion and exclusion criteria are shown in the flow diagram, $\odot$ Fig. 1.

Undetermined FLLs in CT were defined as lesions, including cysts in the liver, not characterised by the imaging modality, requiring further imaging, histology or clinical follow-up.

\section{Diagnostic materials and equipment}

All patients with CT-undetermined FLLs were examined at the same centre with multi-slice scanners (Brilliance CT 64-channel, Philips, The Netherlands). All ultrasound and CEUS examinations were performed by one of the 2 identical high-end scanners LOGIQ E9 (General Electric Healthcare, USA) with

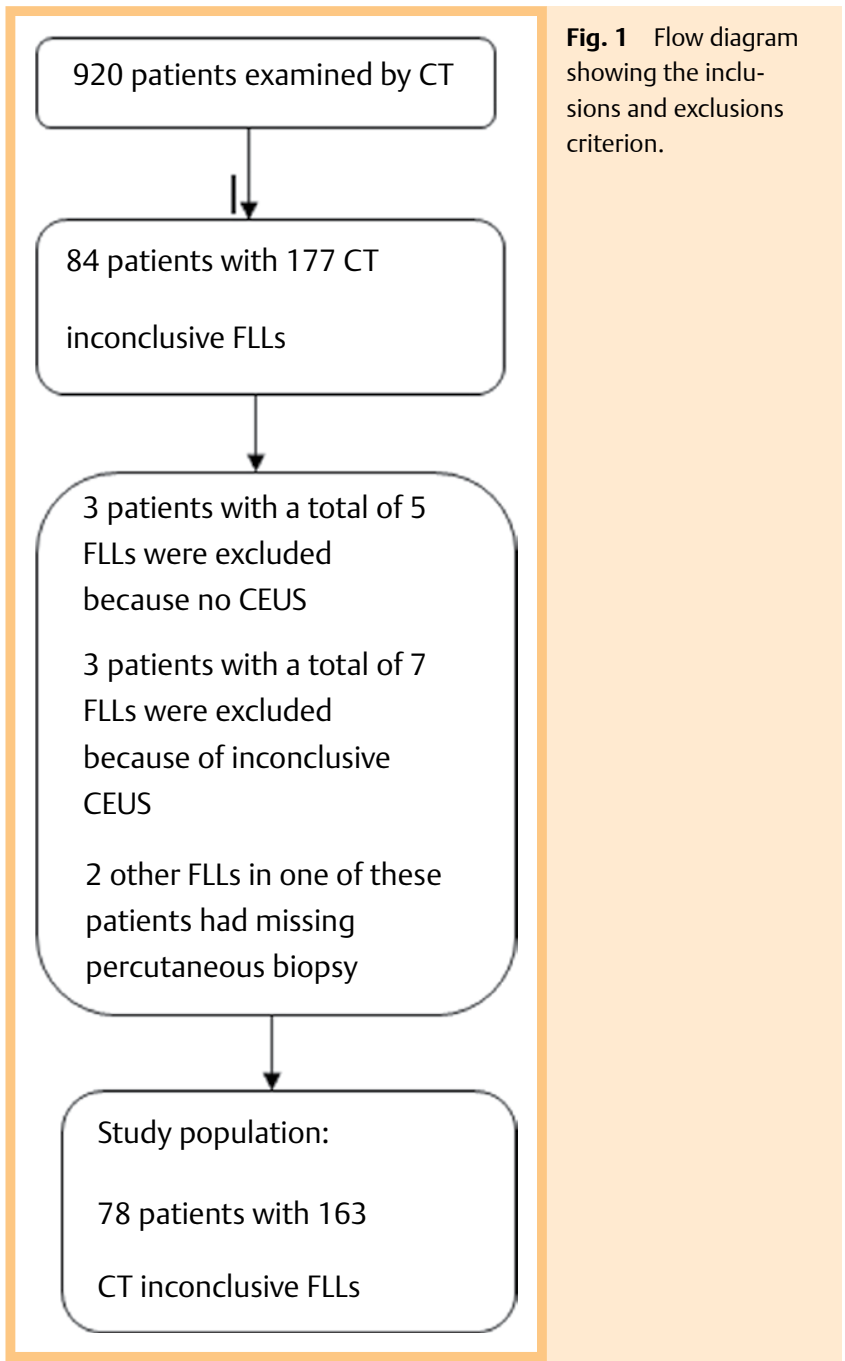

contrast-specific software, using pulse inversion harmonic imaging, with low mechanical index (MI). SonoVue (Bracco Imaging, Italy) was used as the ultrasound contrast medium. SonoVue, which is a second generation contrast medium, consists of stabilised micro bubbles of sulphur hexafluoride with a diameter of $2.5 \mu \mathrm{m}$, in saline solution [14].

\section{Contrast-enhanced ultrasound}

All patients referred to CEUS were scanned with B-mode ultrasound to localise the FLLs. Intravenous access was done using a winged infusion set where a $1.2 \mathrm{ml}$ bolus of SonoVue was injected, followed by $5 \mathrm{ml}$ of saline solution. With the FLLs in the field of view, the vascular distribution of SonoVue in the arterial, portal venous phases and the late phase was recorded in $15 \mathrm{~s}$ cine clips. The different phases were defined as follows:

- Arterial phase: approx. 10-35s after bolus

- Portal venous phase: approx. 35-120 s after bolus

- The late phase: $>120$ s after bolus

5 radiologists performed the CEUS examinations. Their experience in performing and interpreting CEUS ranged from less than one and up to 6 years of experience, and when needed, the less experienced radiologist was supervised by the more experienced. The CEUS examinations were interpreted primarily at the bedside and, when needed, at PACS work stations. This study did not distinguish among the different radiologists and their level of experience. 
Table 1 Diagnostic criteria of CEUS for typical malignant FLLs in non-cirrhotic liver according to EFSUMB guidelines.

\begin{tabular}{llll} 
FLL & Arterial phase & Portal phase & Late phase \\
HCC & Hyper-enhancing & Iso-enhancing & Hypo-enhancing/Slight or moderate wash-out \\
\hline Metastasis & Rim-enhancement & Hypo-enhancing/Wash-out & Hypo-enhancing/Wash-out \\
Metastasis & Enhancement & Hypo-enhancing/Wash-out & Hypo-enhancing/Wash-out \\
\hline Cholangiocarcinoma & Rim-enhancement & Hypo-enhancing/Wash-out & Hypo-enhancing/Wash-out
\end{tabular}

Table 2 Diagnostic criteria of CEUS for typical benign FLLs in non-cirrhotic liver according to EFSUMB guidelines.

\begin{tabular}{|lll}
\hline FLL & Arterial phase & Portal venous phase \\
\hline Hemangioma & Peripheral-nodular or no central enhancement & Partial or complete centripetal filling Complete enhancement \\
\hline FNH & Early hyper-enhancing, spoke-wheel pattern & Hyper-enhancing \\
\hline Focal fatty infiltration/Sparing & Iso-enhancing & Iso-enhancing \\
\hline Simple cyst & Non-enhancing & Non-enhancing \\
\hline $\begin{array}{l}\text { Adenoma } \\
\text { Abscess }\end{array}$ & Hyper-enhancing & Hyper-enhancing \\
\hline
\end{tabular}

Table 3 Distribution of benign and malignant FLLs as found by the standards of reference.

\begin{tabular}{lccc} 
Standard of Reference & Malignant & Benign & FLLs \\
Clinical follow-up & 4 & 65 & $69(42.33 \%)$ \\
\hline CT & 10 & 51 & $61(37.42 \%)$ \\
Histopathology & 3 & 4 & $7(4.29 \%)$ \\
\hline Ultrasound & 0 & 1 & $1(0.61 \%)$ \\
\hline CEUS & 0 & 24 & $24(14.72 \%)$ \\
\hline PET/CT & 1 & 0 & $1(0.61 \%)$ \\
\hline Total & 18 & 145 & $163(100.00 \%)$
\end{tabular}

\section{Diagnostic criteria for CEUS}

The radiologists' final written report on CEUS examinations was used to establish the diagnosis of the FLLs. The diagnoses of the FLLs by CEUS were based on vascular patterns of SonoVue in different phases, also known as wash-in and wash-out, in contrast to adjacent normal liver parenchyma, as defined by Guidelines and Good Clinical Practice Recommendations for ContrastEnhanced Ultrasound [8], see $\bullet$ Tables 1 and $\bullet 2$. FLLs with a wash-out pattern in the portal venous phase and the late phase were interpreted as malignant. FLLs with no wash-out patterns were interpreted as benign.

\section{Reference standard}

Histological data for the 78 included patients were searched to establish the final diagnosis of the 163 FLLs. Histological data from percutaneous biopsy or surgical resection were considered the gold standard in 7 cases. When histology was not available, imaging and one-year clinical follow-up were used in 156 cases. Imaging follow-up was performed using CT and PET/CT, whereas CEUS was used only for benign lesions. None of the malignant FLLs had ultrasound or CEUS as the standard of reference, see $\odot$ Table 3. The reports in RIS/PACS, written by radiologists, were used for imaging follow-up. Clinical follow-up was established using the hospital electronic medical records. In this study, the reference standard was composite, consisting of histology from percutaneous biopsy or surgical resection, imaging, and clinical follow-up. $\odot$ Fig. 2 shows the work flow diagram for establishing the reference standard.

\section{Statistical analysis}

Data were analysed descriptively according to data type: categorical variables were displayed by frequencies and respective percentages, continuous variables by means and standard deviations or medians and ranges, depending on whether continuous variables were roughly symmetrically distributed as judged visually by means of histograms and approximating normal distributions. The diagnostic accuracy of CEUS was calculated on a per-lesion basis. Sensitivity, specificity, positive predictive value (PPV) and negative predictive value (NPV) were calculated with 95\% confidence intervals (95\% CI) using standard errors allowing for intragroup correlation due to the clustered structure of FLLs. Malignant and benign lesions, which were classified correctly compared to the composite reference standard, were assessed as true positive and true negative, respectively. Lesions, which were inconclusive according to CEUS, were left out of the primary analyses. In sensitivity analyses, we studied 2 strategies: (a) classifying those inconclusive lesions in case of malignancy as false negative and benignity as false positive (worst case scenario); (b) classifying all lesions which were inconclusive according to CEUS as positive (clinically conservative strategy) [15]. Statistical analyses were done using Stata IC 14.1 (StataCorp. LP, College Station, TX 77845, USA).

\section{Results \\ $\nabla$}

We included 78 patients with 163 undetermined FLLs in this study. Multiple FLLs in a patient were counted as 5 FLLs, because the exact numbers of FLLs in these patients were not available. In 78 patients, $18 \%$ had multiple FLLs, $1 \%$ had 4 FLLs, $8 \%$ had 3 FLLs, $18 \%$ had 2 FLLs and 55\% had a single FLL. The 163 CT-undetermined FLLs were examined by CEUS and compared with the reference standard. CEUS found 17 true positive, 144 true negative, one false positive and one false negative FLL. CEUS were inconclusive for 7 FLLs in 3 patients. 2 of these patients had one FLL each and one had 5 FLLs.

All malignant FLLs, in 78 patients, were confirmed by the standard of reference as liver metastases. 9 metastases were found in 7 colorectal cancer patients. 3 metastases were found in one patient with uterine cancer. One metastasis was found in one lung cancer patient. 5 metastases were found in one patient diagnosed with prostate cancer. In the 3 cases where CEUS was inconclusive, one patient had one FNH determined by CT. One patient had one metastasis and one had 5 metastases determined by histology. The most frequent benign FLLs were cysts. CEUS was unable to establish the differential diagnoses for the 


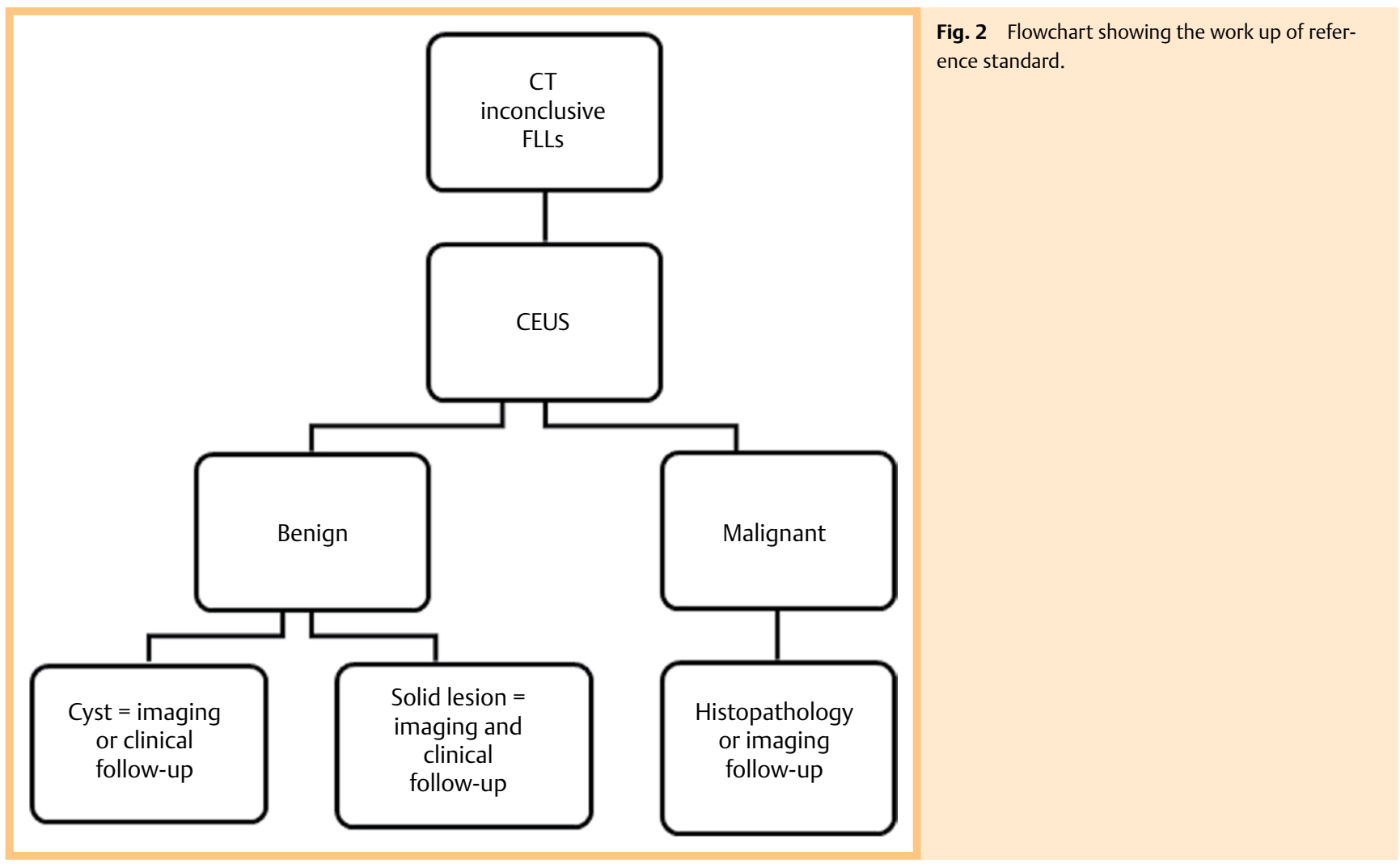

remaining 40 benign FLLs, see $\bullet$ Table 4. The 78 included patients had 163 FLLs, mean size $1.1 \mathrm{~cm}$; range $0.1-5.2 \mathrm{~cm}$, SD $0.73 \mathrm{~cm}$. CEUS found 17 true positive FLLs, with a mean size of $1.3 \mathrm{~cm}$; range $0.8-1.8 \mathrm{~cm}, \mathrm{SD} 0.39 \mathrm{~cm}$. The 144 true negative FLLs found by CEUS had a mean size of $1.1 \mathrm{~cm}$, range $0.1-5.2 \mathrm{~cm}$, SD $0.79 \mathrm{~cm}$. One $1.5 \mathrm{~cm}$ FLL was false positively diagnosed by CEUS as a metastasis. This FLL was diagnosed by histology as biliary hamartoma (von Meyenburg complex). One $0.7 \mathrm{~cm}$ FLL was wrongly diagnosed by CEUS as benign non-specific, but imaging follow-up done by PET/CT, found this FLL to be a metastasis. Sensitivity, specificity, PPV, NPV and accuracy with 95\% CI of CEUS were 94.4\% (95\% CI: 56.3-99.5\%), 99.3\% (95\% CI: 94.999.9\%), 94.4\% (95\% CI: 56.3-99.5\%), 99.3\% (95\% CI: 94.9-99.9\%), and $98.7 \%$ (95\% CI: 94.9-99.7\%), respectively, see $\bullet$ Table 5. Sensitivity analyses (a) where classifying 3 inconclusive lesions in case of malignancy as false negative and benignity as false positive are shown in 0 Table 6; these indicated a significant drop in sensitivity and PPV from 94.4 to $70.8 \%$ and from 99.3 to $89.5 \%$, respectively. Sensitivity analyses (b) classifying all lesions that were inconclusive according to CEUS as positive are shown in $\odot$ Table 7, suggesting a smaller increase in sensitivity by $1.4 \%$ and a decrease in PPV by $2.4 \%$. No side effects were reported from the use of SonoVue.

\section{Discussion}

$\nabla$

The primary analyses in this study showed a high diagnostic accuracy for CEUS in distinguishing between malignant and benign FLLs, which were undetermined by CT. The results of our study showed that CEUS was capable of correctly diagnosing 17 malignant FLLs among 18 with a relatively narrow 95\% CI. Our study also showed that CEUS was capable of correctly diagnosing 144 FLLs from 145 benign FLLs with a very narrow 95\% CI.
Table 4 Differential diagnoses of the FLLs as found by the standards of reference.

$\begin{array}{lc}\text { FLL } & \text { Frequnecy } \\ \text { Cyst } & 64(39.26 \%) \\ \text { Hemangioma } & 36(22.09 \%) \\ \text { Metastasis } & 18(11.04 \%) \\ \text { FNH } & 1(0.61 \%) \\ \text { Biliary hamartoma } & 1(0.61 \%) \\ \text { Focal fatty infiltration } & 3(1.84 \%) \\ \text { Benign non-specific } & 40(24.54 \%) \\ \text { Total } & 163(100.00 \%)\end{array}$

Table 5 Primary analysis: sensitivity, specificity, PPV, NPV, accuracy and $95 \% \mathrm{Cl}$ of CEUS in CT undetermined FLLS.

\begin{tabular}{lcc} 
Parameter & Point Estimate & $\mathbf{9 5 \% ~ C l}$ \\
Sensitivity & $94.4 \%$ & $56.3-99.5 \%$ \\
\hline Specificity & $99.3 \%$ & $94.9-99.9 \%$ \\
\hline PPV & $94.4 \%$ & $56.3-99.5 \%$ \\
\hline NPV & $99.3 \%$ & $94.9-99.9 \%$ \\
\hline Accuracy & $98.7 \%$ & $94.9-99.7 \%$
\end{tabular}

Table 6 Sensitivity analysis (a) including the inconclusive CEUS results classifying 6 malignant lesions and one benign lesion in 3 patients as false negative and false positive, respectively. Sensitivity, specificity, PPV, NPV, accuracy and $95 \% \mathrm{Cl}$ of CEUS in CT-undetermined FLLs.

\begin{tabular}{lcc} 
Parameter & Point Estimate & $\mathbf{9 5 \%} \mathbf{C l}$ \\
Sensitivity & $70.8 \%$ & $25.7-94.5 \%$ \\
\hline Specificity & $98.6 \%$ & $94.4-99.7 \%$ \\
\hline PPV & $89.5 \%$ & $55.7-98.3 \%$ \\
\hline NPV & $95.4 \%$ & $81.8-98.9 \%$ \\
\hline Accuracy & $94.7 \%$ & $83.9-98.4 \%$
\end{tabular}


The $0.7 \mathrm{~cm}$ false negative FLL was diagnosed by PET/CT as a metastasis and was located in segment VI of the liver. It is difficult to determine the exact cause of the false negative CEUS finding. It is well known that ultrasound is operator-dependent. Therefore, it is possible that the false negative FLL is due to inadequate visualisation of the segment VI of the liver.

CEUS uses the same contrast dynamics as CT, requires fewer resources and does not use ionizing radiation. Unlike iodinated CT contrast agents, SonoVue remains in the blood vessels, due to the size of the microbubbles [6,7]. This true blood pool characteristic combined with the dynamic picture acquisition and harmonic imaging technique makes CEUS capable of detecting even the smallest amount of contrast agent in the capillaries of the FLLs, in the arterial, portal venous and late venous phases. Single phase CT is inadequate in visualising the 3 perfusion phases. CT can have difficulties visualising the tumour perfusion of the FLLS due to incorrect timing between contrast injection and picture acquisition [6,7]. Small FLLs $\leq 2 \mathrm{~cm}$ are often difficult to diagnose correctly by CT and are described by radiologists as undetermined. It is commonly known that these difficulties are caused by partial volume averaging, which makes correct measurements of Hounsfield units difficult [12]. The results of this study showed that most FLLs were $\leq 2 \mathrm{~cm}$, in accordance with the lit-

Table 7 Sensitivity analysis (b) including the inconclusive CEUS results classifying 7 lesions in 3 patients as positive. Sensitivity, specificity, PPV, NPV, accuracy and $95 \% \mathrm{Cl}$ of CEUS in CT-undetermined FLLs.

\begin{tabular}{|c|c|c|}
\hline Parameter & Point Estimate & $95 \% \mathrm{Cl}$ \\
\hline Sensitivity & $95.8 \%$ & $66.4-99.6 \%$ \\
\hline Specificity & $98.6 \%$ & $94.4-99.7 \%$ \\
\hline PPV & $92.0 \%$ & $65.1-98.6 \%$ \\
\hline NPV & $99.3 \%$ & $95.0-99.9 \%$ \\
\hline Accuracy & $98.2 \%$ & $94.4-99.5 \%$ \\
\hline
\end{tabular}

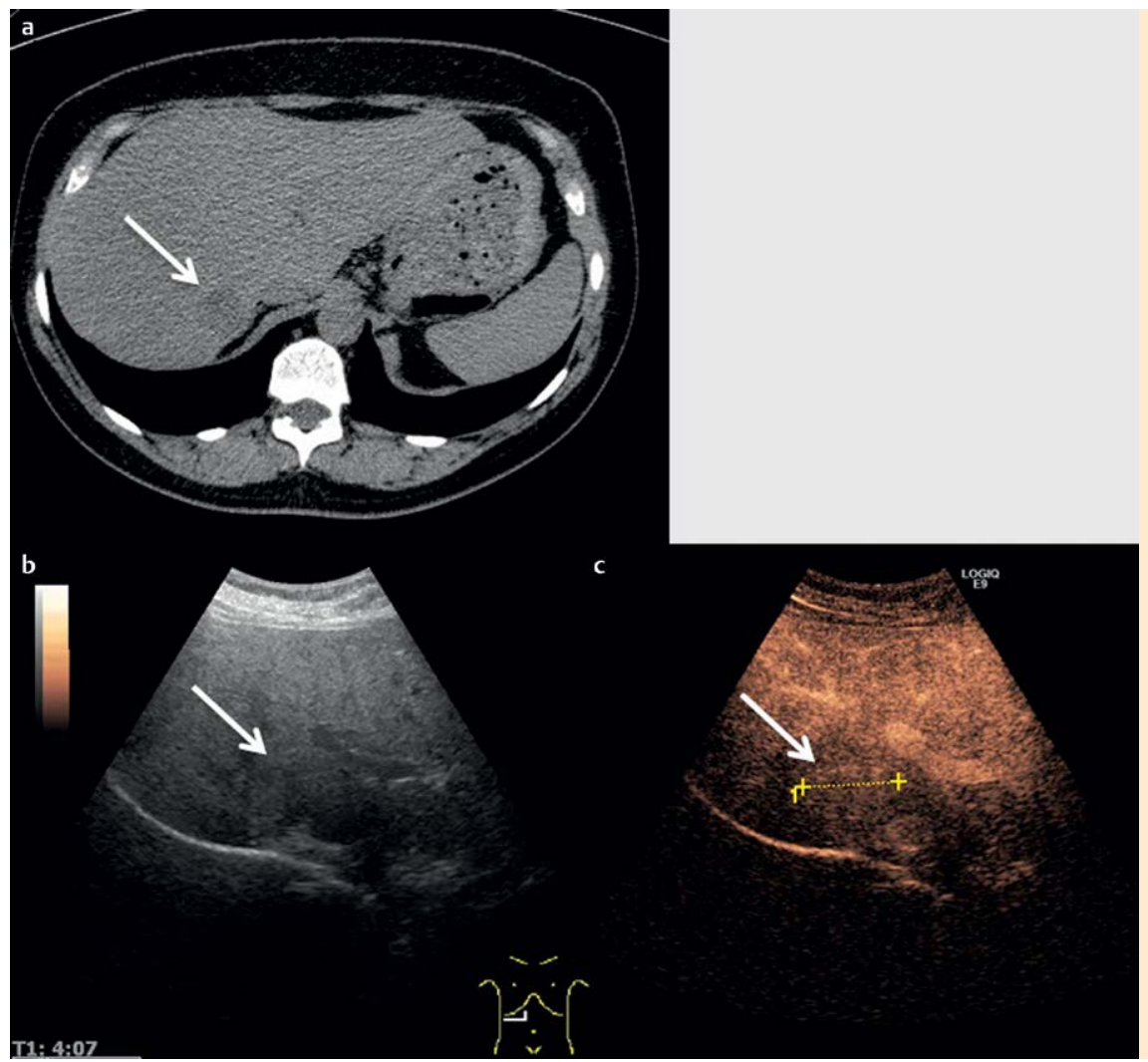

erature. Differentiation of FLLs with CEUS are based on uptake and wash-out patterns compared with the normal liver parenchyma, see $\odot$ Tables 1, 2 and the portal and late venous phases are especially important for the differentiation between malignant and benign lesions [7-11]. The minute differences between the different FLLs in the arterial phase make their differentiation somewhat difficult, and the differentiation is dependent on the experience of the ultrasound operator. However, the most important criteria for distinguishing between malignant and benign FLLs are the portal and late venous phases, where the malignant FLLs have a wash-out pattern and the benign FLLs are hyper/isoechoic, see $\odot$ Fig. 3, 4. The high sensitivity of ultrasound with only a small amount of contrast agent is mainly due to high spatial resolution of ultrasound, combined with a high contrast resolution from the microbubbles non-linear harmonic frequencies and high temporal resolution [16]. Contrast agents like SonoVue are non-nephrotoxic and considered safe with few side effects [8]. Patients referred to CEUS with CT-undetermined FLLs are often fearful of malignancy. The interpretation method of CEUS, which is often performed at the bedside, makes fast and accurate diagnoses possible, so that patients with benign FLLs can be excluded, avoiding unnecessary and demanding ionizing radiological follow-up, biopsy, and consequent complications. Previous studies have shown that CEUS may have a high diagnostic value in differentiating FLLs [7,9,10,17-19]. In a recently published study, CEUS showed a high diagnostic accuracy in CTundetermined FLLs [20]. The results of our study, however, are not directly comparable with these studies. The FLLs in our study were all undetermined at the initial CT. Unlike other studies, we included cysts, focal fatty infiltrations, focal fatty sparings and hemangiomas in order to better estimate the overall diagnostic accuracy of CEUS in clinical practice. Our study includes more patients with more undetermined FLLs than previous studies. The diagnostic method of CEUS in our study was

Fig. 3 a CT scan with undetermined FLL. b Same FLL on B-mode ultrasound. c Same FLL on CEUS with isoenhancing contrast pattern in late venous phase. The FLL was benign and characterised as $\mathrm{FNH}$ 


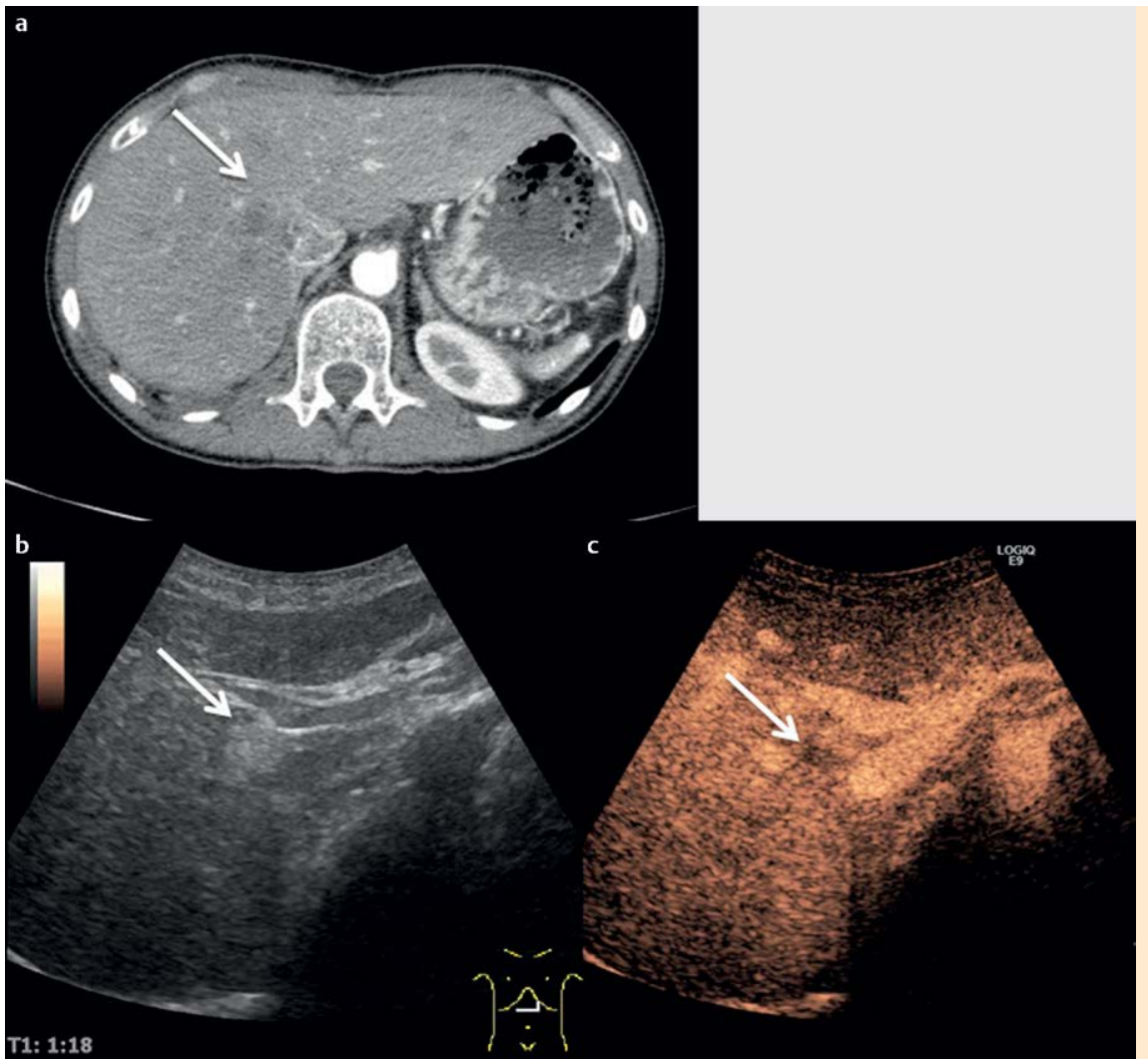

Fig. 4 a CT scan with undetermined focal liver lesion (FLL). b Same FLL on B-mode ultrasound. c Same FLL on contrast enhanced ultrasound (CEUS) with washout pattern in late venous phase. The FLL was malignant and characterised as a metastasis.

performed at the bedside with cine loops option, for on-site review and consultation with more experienced radiologists. We believe that the reading method in our study, which also allows for multiple contrast agent administrations, can improve the overall diagnostic accuracy of CEUS in CT-undetermined FLLs. The primary analyses of our study have some limitations regarding the diagnostic value of CEUS: the exclusion of CEUS-inconclusive FLLs has artificially increased the sensitivity and PPV, whereas sensitivity analyses (a) have decreased the values considerably. We believe that approach (a) is too conservative and that analyses (b) are more useful in clinical practice as inconclusive CEUS is preferably assumed to indicate malignancy in order to proceed with percutaneous biopsy. Furthermore, our study also showed that, if a CT-undetermined FLL is diagnosed by CEUS as benign, it is safe to consider it as such. Our study has limitations regarding the standard of reference. Histological references were only available for 7 CEUS-conclusive FLLs, thus most FLLs were confirmed by clinical follow-up or imaging follow-up like CT, ultrasound, CEUS and PET/CT. It can be poor research practice to use an initially inconclusive modality like CT or ultrasound/ CEUS as a standard of reference. We believe that this approach is useful because if a FLL has not grown in size for at least one year, it is safe to consider it as benign. A study from Tan et al. also used a one-year follow-up and if a liver lesion remained stable, the lesion was considered benign [21].

Furthermore, ultrasound/CEUS was not used as its own standard of reference for any malignant FLLs. The use of CT for imaging follow-up indicates that most patients with malignant FLLs were known to have a malignant condition. In our study, we did not distinguish between the experience of the radiologists who interpreted the CEUS studies, preventing us from examining inter-rater variations. The diagnostic accuracy is higher in our study because all CEUS studies were interpreted via consensus with the most experienced radiologist. This study had some minor limitations regarding the population because we did not consider the inclusion or distribution of symptomatic vs. asymptomatic patients. The low prevalence of malignant FLLs can be interpreted as a low prevalence of symptomatic patients in our study population. Patients with several and multiple FLLs were regarded as having 5 FLLs, because the exact number of FLLs per patient was not available from the CT interpretations. This approach must have reduced the number of benign FLLs. In our study, we excluded 2 FLLs in one patient due to lack of biopsy. Considering the false positive nature of the remaining FLL in one patient, it can be argued that this approach has increased the specificity and NPV of CEUS. The final diagnosis of the remaining FLL, using histology, was biliary hamartoma, see $\bullet$ Fig. 5. Few case studies exist regarding the CEUS characteristics of biliary hamartoma, and there seem to be contradictory reports regarding the portal and late venous phases $[17,22,23]$. The diagnostic values of CEUS in our study were calculated on a lesion by lesion basis and therefore cannot be directly transferred to a patientby-patient basis in clinical practice.

Even though our study has some limitations, CEUS showed a high diagnostic accuracy in distinguishing between malignant and benign FLLs that were undetermined at the initial CT. Therefore, we cautiously conclude that the results of this study are useful in clinical practice.

\section{Affiliations}

${ }^{1}$ Health and Technology, Metropolitan University College, Copenhagen N, Denmark

2Department of Diagnostic Imaging, Roskilde Sygehus, Roskilde, Denmark ${ }^{3}$ Department of Nuclear Medicine, Odense University Hospital, Centre of Health Economics Research, University of Southern Denmark, Odense, Denmark ${ }^{4}$ Department of Radiology, Vejle Hospital, University of Southern Denmark, Vejle, Denmark 


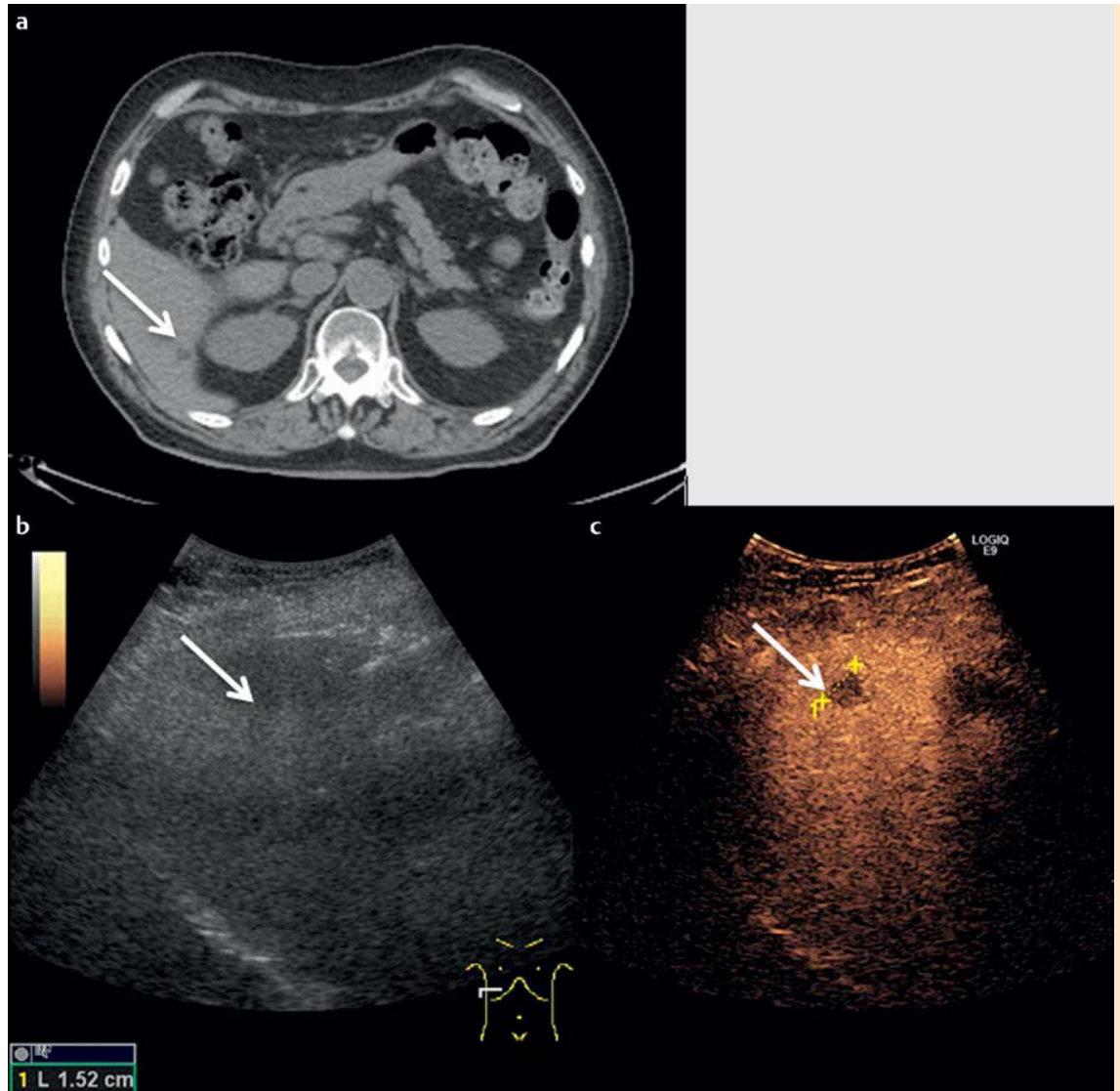

Fig. 5 a CT scan with undetermined focal liver lesion (FLL). b Same FLL on B-mode ultrasound. c Same FLL on contrast enhanced ultrasound (CEUS) with washout pattern in portal and late venous phase, wrongly diagnosed by CEUS as metastasis. Histopathology showed biliary hamartoma.

\section{References}

1 Volk M, Strotzer M, Lenhart M, Techert J, Seitz J, Feuerbach S. Frequency of benign hepatic lesions incidentally detected with contrast-enhanced thin-section portal venous phase spiral CT. Acta Radiol 2001; 42: 172-175

2 Hohmann J, Skrok J, Basilico R, Jennett M, Müller A, Wolf KJ et al. Characterisation of focal liver lesions with unenhanced and contrast enhanced low MI real time ultrasound: on-site unblinded versus offsite blinded reading. Eur J Radiol 2012; 81: e317-e324

3 Sacks A, Peller PJ, Surasi DS, Chatburn L, Mercier G, Subramaniam RM. Value of PET/CT in the management of liver metastases, part 1. AJR Am J Roentgenol 2011; 197: W256-W259

4 Albrecht T, Hohmann J, Oldenburg A, Skrok J, Wolf KJ. Detection and characterisation of liver metastases. Eur Radiol 2004; 14: (Suppl 8): P25-P33

5 Bleuzen A. Tranquart $F$. Incidental liver lesions: diagnostic value of cadence contrast pulse sequencing (CPS) and SonoVue. Eur Radiol 2004; 14: (Suppl 8): P53-P62

6 Westwood M, Joore M, Grutters J, Redekop K, Armstrong N, Lee K et al. Contrast-enhanced ultrasound using SonoVue(R) (sulphur hexafluoride microbubbles) compared with contrast-enhanced computed tomography and contrast-enhanced magnetic resonance imaging for the characterisation of focal liver lesions and detection of liver metastases: a systematic review and cost-effectiveness analysis. Health Technol Assess 2013; 17: 1-243

7 Quaia E, Calliada F, Bertolotto M, Rossi S, Garioni L, Rosa L et al. Characterization of focal liver lesions with contrast-specific US modes and a sulfur hexafluoride-filled microbubble contrast agent: diagnostic performance and confidence. Radiology 2004; 232: 420-430

8 Claudon M, Dietrich CF, Choi BI, Cosgrove DO, Kudo M, Nolsøe CP et al. Guidelines and Good Clinical Practice Recommendations for Contrast Enhanced Ultrasound (CEUS) in the Liver - Update 2012. Ultraschall Med 2013; 34: 11-29

9 Dai Y, Chen MH, Yin SS, Yan K, Fan ZH, Wu W et al. Focal liver lesions: can SonoVue-enhanced ultrasound be used to differentiate malignant from benign lesions? Invest Radiol 2007; 42 (8): 596-603

10 von Herbay A, Westendorff J, Gregor $M$. Contrast-enhanced ultrasound with SonoVue: differentiation between benign and malignant focal liver lesions in 317 patients. J Clin Ultrasound 2010; 38: 1-9

11 Ooi CC, Low SC, Schneider-Kolsky M, Lombardo P, Lim SY, Abu Bakar $R$ et al. Diagnostic accuracy of contrast-enhanced ultrasound in differentiating benign and malignant focal liver lesions: a retrospective study. J Med Imaging Radiat Oncol 2010; 54: 421-430
12 Laghi F, Catalano O, Maresca M, Sandomenico F, Siani A. Indeterminate, subcentimetric focal liver lesions in cancer patients: additional role of contrast-enhanced ultrasound. Ultraschall in Med 2010; 31: 283-288

13 Bossuyt PM, Reitsma JB, Bruns DE, Gatsonis CA, Glasziou PP, Irwig LM et al. Towards complete and accurate reporting of studies of diagnostic accuracy: The STARD Initiative. Radiology 2003; 226: 24-28

14 Correas JM, Bridal L, Lesavre A, Mejean A, Claudon M, Helenon O. Ultrasound contrast agents: properties, principles of action, tolerance, and artifacts. Eur Radiol 2001; 11: 1316-1328

15 Shinkins B, Thompson M, Mallett S, Perera R. Diagnostic accuracy studies: how to report and analyse inconclusive test results. Bmj 2013; 346: f2778

16 Quaia E. Solid focal liver lesions indeterminate by contrast-enhanced CT or MR imaging: the added diagnostic value of contrast-enhanced ultrasound. Abdom Imaging 2012; 37: 580-590

17 Berry JD, Boxer ME, Rashid HI, Sidhu PS. Case report: microbubble contrast-enhanced ultrasound characteristics of multiple biliary hamartomas (von Meyenberg Complexes). Ultrasound 2004; 12: 95-97

18 Beaton C, Cochlin D, Kumar N. Contrast enhanced ultrasound should be the initial radiological investigation to characterise focal liver lesions. Eur J Surg Oncol 2010; 36: 43-46

19 Sporea I, Badea R, Popescu A, Sparchez Z, Sirli RL, Danila M et al. Contrast-enhanced ultrasound (CEUS) for the evaluation of focal liver lesions - a prospective multicenter study of its usefulness in clinical practice. Ultraschall in Med 2014; 35: 259-266

20 Quaia E, De Paoli L, Angileri R, Cabibbo B, Cova MA. Indeterminate solid hepatic lesions identified on non-diagnostic contrast-enhanced computed tomography: assessment of the additional diagnostic value of contrast-enhanced ultrasound in the non-cirrhotic liver. Eur J Radiol 2014; 83: 456-462

21 Tan CH, Bhosale PR, Das P, Crane CH, Viswanathan C, Raval B et al. Multidetector computed tomography follow-up of hypoattenuating small liver lesions in patients with rectal cancer. Am J Clin Oncol 2011; 34: 411-416

22 Lung PF, Jaffer OS, Akbar N, Sidhu PS, Ryan SM. Appearances of von meyenburg complex on cross sectional imaging. Journal of clinical imaging science 2013; 3: 22

23 Hohmann J, Loddenkemper C, Albrecht T. Assessment of a biliary hamartoma with contrast-enhanced sonography using two different contrast agents. Ultraschall in Med 2009; 30: 185-188 